\title{
Combining Ability for Yield and Quality Related Traits in Tomato (Solanum lycopersicum L.)
}

\author{
Rakesh Kumar Meena* and Sanjay Kumar \\ Department of Horticulture, Babasaheb Bhimrao Ambedkar University, \\ Vidya-Vihar, Rae Bareli Road, Lucknow-226025, India \\ *Corresponding author
}

\begin{tabular}{l}
\hline \\
\hline Ke y w o r d s \\
Combining ability, \\
Growth, Yield, \\
Quality and Tomato \\
\hline Article Info \\
\hline $\begin{array}{l}\text { Accepted: } \\
\text { 23 April } 2020 \\
\text { Available Online: } \\
\text { 10 May } 2020\end{array}$ \\
\hline
\end{tabular}

A B S T R A C T

Thirty six hybrids generated from crossing twelve lines with three testers were studied along with parents for combining ability in tomato. The specific combining ability (SCA) was significant for all the characters, indicating the importance of both additive and non-additive genetic components. But it is found that there was predominance of non-additive genetic components for expression of different traits in the present set of materials. The most promising specific combiner for fruits per plant and pericarp thickness was $\mathrm{H}-86$ x Kashi Amrit. Other cross combiner FLA7171 x Kashi Amrit best in locules per plant and Vitamin C .Hence, the present study was carried out to obtain information on combining ability involved in expressing the different characters in tomato.

\section{Introduction}

Tomato [Solanum lycopersicon L. isone of the most important commercial crops grown extensively in the tropical and sub-tropical region of the world. It ranks second only after potato (Bose et al., 2). Tomato (Solanum lycopersicum L.) belongs to Solanaceae family having chromosome number $2 n=2 x=24$. It has originated from wild form in the Peru- Equador Bolivia region of South America (Rick, 7). It has commercial value in the extraction of tomatine, a steroidal hormone, which is used as a substitute of diosgenin (Amid et al., 1). The unripe green fruits are used for making pickles, preserves and are consumed after cooking as vegetable (Kaur et al., 5).

Tomato is a rich source of antioxidants (mainly lycopene and $\beta$-carotene), Vitamin A, Vitamin $\mathrm{C}$ and minerals like $\mathrm{Ca}, \mathrm{P}$ and $\mathrm{Fe}$. In tomato total antioxidant capacity ranges from 80 to $200 \mu \mathrm{mol}$. Ascorbic acid contents of 
tomatoes have been found to vary according to color and it ranged from 23.21- 40.44 and 24.38 - $33.87 \mathrm{mg} / 100 \mathrm{~g}$ in red and yellow cultivars, respectively (Singh et al., 8).

\section{Materials and Methods}

Twelve genetically diverse germplasm lines of tomato viz., IIVR-Sel.-1, G-3, S. Naveen, DVRT-2, H-24, H-86, H-88, Pusa Sheetal, FLA 7171, Hisar Arun, Sel.-32 and Flora Dode were used as a lines and three (Pusa Sadabahar, Kashi Vishesh and Kashi Amrit were crossed with three testers (Pusa Sadabahar, Kashi Vishesh and Kashi Amrit). The resulting $36 \mathrm{~F}_{1} \mathrm{~s}$ along with 12 lines and three testers were evaluated in randomized block design (RBD) with three replications at Horticulture Research Farm, Department of Applied Plant Science (Horticulture), Babasaheb Bhimrao Ambedkar University, Vidya- Vihar, Rea Bareli Road, Lucknow during the year 2014-15.

Sixteen plants of each genotypes were transplanted at a spacing of $60 \mathrm{~cm} \mathrm{x} 45 \mathrm{~cm}$. Observations were recorded like plant height (cm), number of branches/plant, days to 50 percent flowering, number of clusters/plant, number of flowers/cluster, number of fruits/cluster, number of fruits/plant, average fruit weight, number of locules/fruit, pericarp thickness, fruit length, fruit width, number of ridges on fruit, fruit yield/plant, TSS ( ${ }^{0}$ brix) and vitamin c $(\mathrm{mg} / 100 \mathrm{~g})$ were recorded.The standard procedures developed (Kempthorne, 6) were followed to estimate the mean sum of squares (MSS) along with variances of SCA and GCA. Standard statistical tools (Singh and Choudhury, 9) were used to analyze thecombining ability effects.

\section{Results and Discussion}

The specific combining ability is represented the dominance and epistatic component of variation that are non-additive gene action. It can be utilized in generation like developing $\mathrm{F}_{1}$ hybrids. In the present investigation, the thirty six cross manifested consistently high specific combining ability effects for most of the characters (Dahiya et al., 4).

In the present study findings (Table 1 and 2) revealed that the significant and desirable cross in order of merit were H-86 x Pusa Sadabahar and S. Naveen x Kashi Vishesh for plant height FLA 7171 x Pusa Sadabahar for Number of branches per plant, H-24 x Kashi Amrit for days to $50 \%$ flowering, S. Naveenx Kashi Vishesh for clusters per plant, FLA $7171 \mathrm{x}$ Pusa Sadabahar for flowers per cluster, FLA 7171 x Pusa Sadabahar for fruits per cluster, H-86 x Kashi Amrit for fruits per plant, H-88 x Kashi Vishesh for average fruit weight, H-24 x Pusa Sadabahar for locules per fruit, IIVR-Sel.-1 x Kashi Amrit for pericarp thickness, S. Naveen x Kashi Amrit for fruit length, H-86 x Pusa Sadabahar for fruit width,G-3 x Kashi Amrit for ridges On fruit, S. Naveenx Kashi Vishesh for fruit yield per plant, G-3 x Kashi Vishesh for TSS and H-88 x Kashi Amrit for vitamin C were showed significant and desirable specific combiner.

Similarly, a critical examination of per se performance of best crosses for sixteen characters also reviled that there is no direct relationship between the per se of the crosses and their parents similar findings have been also reported (Brar et al., 3). 
Table.1 Estimation of specific combining ability (SCA) effect for the 8 characters of tomato

\begin{tabular}{|c|c|c|c|c|c|c|c|c|c|}
\hline $\begin{array}{l}\text { S. } \\
\text { No. }\end{array}$ & Crosses & 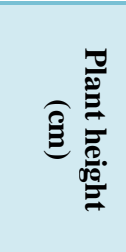 & 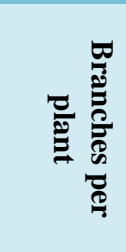 & 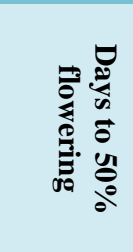 & 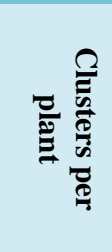 & $\frac{\varrho}{\frac{0}{0}}$ & 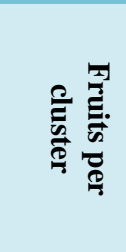 & 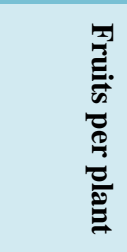 & 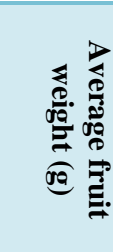 \\
\hline 1 & IIVR-Sel.-1 x Pusa Sadabahar & -0.43 & 0.50 & -0.25 & -0.14 & 0.07 & $-0.40 *$ & 0.26 & -2.30 \\
\hline 2 & IIVR-Sel. 1 x Kashi Vishesh & 0.88 & -0.23 & -0.69 & 0.32 & 0.58 & $0.47^{*}$ & $-3.20 * *$ & -3.87 \\
\hline 3 & IIVR-Sel.-1 x Kashi Amrit & $-0.45 *$ & -0.27 & 0.94 & -0.19 & -0.65 & -0.07 & $2.94 * *$ & $6.17 *$ \\
\hline 4 & G-3 x Pusa Sadabahar & -0.64 & -0.29 & -0.63 & 0.10 & -0.31 & -0.21 & $5.01 * *$ & -3.46 \\
\hline 5 & G-3 x Kashi Vishesh & 0.51 & -0.01 & -0.12 & -0.21 & 0.15 & 0.07 & -1.71 & 1.09 \\
\hline 6 & G-3 x Kashi Amrit & $0.13 *$ & 0.29 & 0.76 & 0.11 & 0.15 & 0.14 & $-3.30 * *$ & 2.37 \\
\hline 7 & S. Naveen x Pusa Sadabahar & -1.46 & $-0.09 *$ & $-1.33^{*}$ & -0.07 & 0.07 & $0.57 * *$ & $-1.81 *$ & 3.98 \\
\hline 8 & S. Naveenx Kashi Vishesh & $1.99 *$ & 0.29 & $1.33^{*}$ & $0.48^{*}$ & 0.10 & -0.01 & $4.19 * *$ & -0.06 \\
\hline 9 & S. Naveen x Kashi Amrit & -0.52 & -0.20 & 0.01 & -0.41 & -0.18 & $-0.56 * *$ & $-2.38 * *$ & -3.92 \\
\hline 10 & DVRT-2 x Pusa Sadabahar & 1.56 & -0.15 & -0.07 & 0.40 & 0.14 & 0.16 & $5.71 * *$ & 3.79 \\
\hline 11 & DVRT-2 x Kashi Vishesh & -1.69 & 0.30 & -0.55 & -0.41 & 0.18 & -0.29 & $-3.85 * *$ & 0.08 \\
\hline 12 & DVRT-2 x Kashi Amrit & 0.13 & -0.15 & 0.63 & 0.01 & -0.32 & 0.12 & $-1.86^{*}$ & -3.87 \\
\hline 13 & H-24 x Pusa Sadabahar & -1.62 & -0.11 & $1.18^{*}$ & -0.23 & -0.10 & 0.30 & $-3.27 * *$ & $5.59 *$ \\
\hline 14 & H-24 x Kashi Vishesh & 0.93 & 0.21 & 0.68 & $0.17 *$ & $-0.71 * *$ & $-0.50^{*}$ & $2.21 *$ & -1.65 \\
\hline 15 & H-24 x Kashi Amrit & 0.69 & -0.10 & $-1.87 * *$ & 0.06 & $0.81 * *$ & 0.20 & 1.07 & -3.95 \\
\hline 16 & H-86 x Pusa Sadabahar & $2.53^{\prime *}$ & 0.17 & 0.24 & -0.03 & -0.36 & -0.18 & $-6.67 * *$ & 4.23 \\
\hline 17 & H-86 x Kashi Vishesh & $-2.32 *$ & 0.14 & 0.27 & -0.23 & 0.02 & 0.35 & 0.53 & 2.41 \\
\hline 18 & H-86 x Kashi Amrit & -0.20 & -0.31 & -0.51 & 0.26 & 0.34 & -0.17 & $6.14 * *$ & $-6.63 * *$ \\
\hline 19 & H-88 x Pusa Sadabahar & 1.61 & 0.05 & 0.86 & -0.12 & -0.05 & 0.14 & $2.54 * *$ & -1.81 \\
\hline 20 & H-88 x Kashi Vishesh & -1.12 & -0.45 & -0.68 & -0.06 & 0.09 & -0.21 & -1.29 & $6.84 * *$ \\
\hline 21 & H-88 x Kashi Amrit & -0.48 & $0.40^{*}$ & -0.18 & 0.18 & -0.04 & 0.07 & -1.25 & -5.03 \\
\hline 22 & Pusa Sheetal x Pusa Sadabahar & -0.81 & -0.01 & 0.51 & -0.44 & -0.11 & -0.11 & 2.01 & -3.04 \\
\hline 23 & Pusa Sheetal x Kashi Vishesh & 0.05 & -0.20 & -0.28 & -0.06 & -0.05 & -0.11 & 1.74 & -2.08 \\
\hline 24 & Pusa Sheetal x Kashi Amrit & 0.76 & 0.21 & -0.23 & $0.50^{*}$ & 0.17 & 0.22 & -3.75 & 5.12 \\
\hline 25 & FLA 7171 x Pusa Sadabahar & 1.26 & $0.36^{*}$ & $-1.18^{*}$ & 0.22 & $1.20 * *$ & $1.13 * *$ & -1.41 & 3.64 \\
\hline 26 & FLA 7171 x Kashi Vishesh & -1.54 & -0.04 & 0.04 & -0.19 & -0.30 & $-0.45^{*}$ & -0.05 & -3.12 \\
\hline 27 & FLA 7171 x Kashi Amrit & 0.29 & $-0.32 *$ & 1.14 & -0.02 & $-0.90 * *$ & $-0.68 * *$ & 1.46 & -0.52 \\
\hline 28 & Hisar Arun x Pusa Sadabahar & -0.57 & -0.22 & $1.26^{*}$ & 0.35 & $-0.54 *$ & -0.52 & -1.91 & -3.04 \\
\hline 29 & Hisar Arun x Kashi Vishesh & 0.38 & -0.14 & -0.54 & -0.06 & -0.09 & 0.34 & 2.07 & 0.42 \\
\hline 30 & Hisar Arun x Kashi Amrit & 0.19 & 0.36 & -0.72 & -0.29 & $0.63 * *$ & 0.18 & -0.16 & 2.62 \\
\hline 31 & Sel.-32 x Pusa Sadabahar & -0.28 & -0.31 & 0.99 & -0.09 & 0.35 & -0.17 & -0.72 & -1.21 \\
\hline 32 & Sel.-32 x Kashi Vishesh & 0.96 & $0.29 *$ & -0.98 & 0.43 & -0.23 & 0.02 & -1.60 & -2.65 \\
\hline 33 & Sel.-32 x Kashi Amrit & -0.68 & $0.02 *$ & -0.01 & -0.34 & -0.12 & 0.15 & 2.32 & 3.87 \\
\hline 34 & Flora Dode x Pusa Sadabahar & -1.14 & 0.09 & $-1.56 * *$ & 0.06 & -0.36 & -0.72 & 0.26 & -6.36 \\
\hline 35 & Flora Dode x Kashi Vishesh & 0.99 & -0.15 & $1.53^{*}$ & $-0.19^{*}$ & 0.27 & 0.32 & 0.97 & 2.59 \\
\hline 36 & Flora Dode x Kashi Amrit & 0.15 & 0.06 & 0.04 & 0.13 & 0.10 & 0.40 & -1.23 & 3.77 \\
\hline \multicolumn{2}{|c|}{$\mathbf{S . E} \pm \mathbf{M}$} & 0.762 & 0.248 & 0.550 & 0.229 & 0.266 & 0.192 & 1.109 & 2.354 \\
\hline \multicolumn{2}{|c|}{ CD (5\%) } & 1.519 & 0.495 & 1.097 & 0.456 & 0.530 & 0.383 & 2.212 & 4.696 \\
\hline
\end{tabular}

*, ** Significant at $5 \%$ and $1 \%$ level, respectively 
Table.2 Estimation of specific combining ability (SCA) effect for the 8 characters of tomato

\begin{tabular}{|c|c|c|c|c|c|c|c|c|c|}
\hline $\begin{array}{c}\text { S. } \\
\text { No. }\end{array}$ & Crosses & 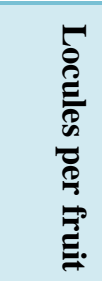 & 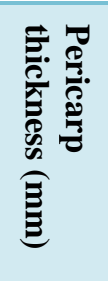 & 疋 & 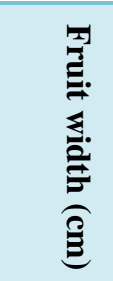 & 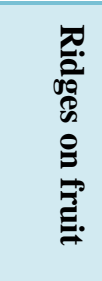 & 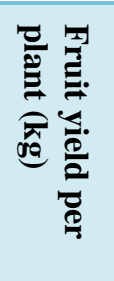 & 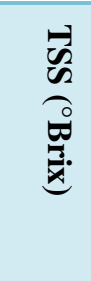 & م \\
\hline 1 & IIVR-Sel.-1 x Pusa Sadabahar & 0.17 & 0.07 & -0.18 & 0.18 & 0.06 & $-0.22 *$ & 0.06 & -0.71 \\
\hline 2 & IIVR-Sel. 1 x Kashi Vishesh & -0.16 & $-0.88 * *$ & -0.08 & -0.34 & 0.01 & $-0.26^{* * *}$ & -0.16 & 1.31 \\
\hline 3 & IIVR-Sel.-1 x Kashi Amrit & -0.02 & $0.81^{* *}$ & 0.26 & 0.16 & -0.07 & $0.48 * *$ & 0.10 & -0.60 \\
\hline 4 & G-3 x Pusa Sadabahar & -0.03 & $-0.44^{*}$ & 0.30 & -0.15 & $-0.42 * *$ & -0.03 & -0.15 & 0.24 \\
\hline 5 & G-3 x Kashi Vishesh & 0.23 & 0.28 & -0.26 & 0.30 & 0.02 & $0.33 * *$ & $0.33^{*}$ & -0.05 \\
\hline 6 & G-3 x Kashi Amrit & -0.20 & 0.16 & -0.04 & -0.15 & $0.40^{* *}$ & $-0.30 * *$ & -0.17 & -0.19 \\
\hline 7 & S. Naveen x Pusa Sadabahar & 0.21 & 0.20 & 0.37 & 0.17 & -0.05 & -0.08 & 0.18 & 0.43 \\
\hline 8 & S. Naveenx Kashi Vishesh & 0.03 & 0.09 & 0.15 & 0.31 & 0.06 & $0.38 * *$ & -0.12 & -0.68 \\
\hline 9 & S. Naveen x Kashi Amrit & -0.23 & -0.30 & $0.52^{*}$ & $-0.49 * *$ & -0.01 & $-0.29 *$ & -0.06 & 0.25 \\
\hline 10 & DVRT-2 x Pusa Sadabahar & -0.19 & 0.25 & $0.33^{*}$ & 0.08 & 0.07 & $0.23 *$ & -0.06 & 0.10 \\
\hline 11 & DVRT-2 x Kashi Vishesh & -0.03 & $-0.35^{*}$ & -0.30 & -0.23 & -0.09 & -0.07 & -0.11 & 1.22 \\
\hline 12 & DVRT-2 x Kashi Amrit & 0.21 & 0.09 & -0.03 & 0.15 & 0.02 & -0.16 & 0.18 & $-1.31 *$ \\
\hline 13 & H-24 x Pusa Sadabahar & $0.80^{* *}$ & 0.27 & -0.09 & 0.21 & 0.07 & $-0.20 *$ & 0.23 & -0.46 \\
\hline 14 & H-24 x Kashi Vishesh & $-0.50 * *$ & -0.16 & -0.09 & -0.10 & $-0.13^{*}$ & -0.02 & -0.25 & -0.85 \\
\hline 15 & H-24 x Kashi Amrit & -0.30 & -0.10 & 0.18 & -0.11 & 0.06 & $0.22 *$ & 0.03 & 1.32 \\
\hline 16 & H-86 x Pusa Sadabahar & -0.31 & 0.17 & 0.16 & $0.38 *$ & $0.31 * *$ & -0.11 & 0.13 & 0.98 \\
\hline 17 & H-86 x Kashi Vishesh & $0.60 * *$ & $0.47 * *$ & $0.17^{*}$ & 0.19 & $-0.16^{*}$ & -0.12 & 0.00 & -0.61 \\
\hline 18 & H-86 x Kashi Amrit & -0.29 & $-0.64 * *$ & -0.33 & 0.18 & $-0.15^{*}$ & $0.22 *$ & -0.13 & -0.37 \\
\hline 19 & H-88 x Pusa Sadabahar & 0.02 & $0.34^{*}$ & -0.05 & -0.15 & 0.06 & 0.03 & 0.02 & -1.70 \\
\hline 20 & H-88 x Kashi Vishesh & -0.21 & $-0.70 * *$ & 0.06 & -0.10 & -0.01 & 0.09 & -0.11 & -0.51 \\
\hline 21 & H-88 x Kashi Amrit & 0.20 & 0.36 & -0.01 & $0.25^{*}$ & -0.05 & -0.12 & 0.09 & $2.22 *$ \\
\hline 22 & Pusa Sheetal x Pusa Sadabahar & -0.54 & -0.23 & -0.63 & -0.02 & 0.07 & -0.02 & -0.13 & -0.78 \\
\hline 23 & Pusa Sheetal x Kashi Vishesh & 0.09 & 0.17 & -0.08 & -0.19 & -0.01 & 0.03 & -0.16 & $2.17 *$ \\
\hline 24 & Pusa Sheetal x Kashi Amrit & 0.45 & 0.06 & 0.71 & 0.20 & -0.07 & -0.01 & $0.29 *$ & -1.39 \\
\hline 25 & FLA 7171 x Pusa Sadabahar & -0.48 & -0.23 & -0.21 & -0.45 & -0.09 & -0.01 & $-0.28 *$ & -0.28 \\
\hline 26 & FLA 7171 x Kashi Vishesh & 0.06 & 0.38 & -0.01 & -0.10 & $0.16^{*}$ & -0.05 & $0.32 *$ & -1.40 \\
\hline 27 & FLA 7171 x Kashi Amrit & $0.42 *$ & -0.15 & 0.22 & $0.55^{*}$ & -0.07 & 0.06 & -0.04 & $1.68 *$ \\
\hline 28 & Hisar Arun x Pusa Sadabahar & -0.13 & -0.39 & 0.01 & 0.14 & 0.03 & $0.21 *$ & -0.20 & 1.75 \\
\hline 29 & Hisar Arun x Kashi Vishesh & 0.13 & 0.27 & 0.06 & 0.36 & -0.01 & -0.10 & 0.16 & -0.54 \\
\hline 30 & Hisar Arun x Kashi Amrit & 0.00 & 0.12 & -0.06 & -0.50 & -0.02 & -0.10 & 0.04 & -1.21 \\
\hline 31 & Sel.-32 x Pusa Sadabahar & 0.57 & -0.23 & 0.00 & 0.45 & -0.08 & 0.08 & 0.14 & -0.15 \\
\hline 32 & Sel.-32 x Kashi Vishesh & -0.36 & 0.32 & -0.03 & 0.05 & 0.10 & -0.17 & 0.07 & 1.48 \\
\hline 33 & Sel.-32 x Kashi Amrit & -0.21 & -0.09 & 0.03 & -0.50 & -0.02 & 0.10 & -0.20 & -1.32 \\
\hline 34 & Flora Dode x Pusa Sadabahar & -0.09 & 0.22 & 0.00 & -0.09 & -0.04 & 0.13 & 0.07 & 0.59 \\
\hline 35 & Flora Dode x Kashi Vishesh & 0.11 & 0.10 & 0.41 & -0.16 & 0.07 & -0.03 & 0.03 & -1.51 \\
\hline 36 & Flora Dode x Kashi Amrit & -0.02 & -0.32 & $-0.41 *$ & 0.25 & -0.03 & -0.10 & -0.10 & 0.93 \\
\hline \multicolumn{2}{|c|}{$\mathbf{S . E} \pm \mathbf{M}$} & 0.189 & 0.129 & 0.148 & 0.166 & 0.077 & 0.060 & 0.145 & 0.714 \\
\hline \multicolumn{2}{|c|}{ CD $(5 \%)$} & 0.376 & 0.258 & 0.296 & 0.331 & 0.153 & 0.120 & 0.289 & 1.424 \\
\hline
\end{tabular}

*,** Significant at 5\% and 1\% level, respectively

\section{Acknowledgments}

I would like to express my very great appreciation to Dr. S. K. Trivedi for his valuable and constructive suggestions during the planning and development of this research work. Mr. Mukesh Kumar for their support in the site measurement.

\section{References}

1. Amid A, Semail S, Jamal P 2011.Tomato leaves methanol extract possesses antiinflammatory activity via inhibition of lipo poly sacharide (LPS)-induced prostaglandin (PGE2). African J. Biotech. 10: 18674-18678. 
2. Bose, T. K., Bose, J., Kabir, T. K., Maity, V. A., Parthasarathy and Som, M. G., 2002. Vegetable crops. Bhumani Mitra Publication, Kolkata, India. Acta Hortic. 37: 77-83.

3. Brar P.S., Singh M. and Gupta R.K. 2005. Combining ability study under high temperature conditions. Haryana $J$. Horti. Sci., 34. 1/2:107- 108.

4. Dahiya M.S., Dhankar B.S. and Pandita H.L. 1985. Line $x$ tester analysis for the study of combining ability in brinjal (Solanum melongena L.). Haryana $J$. Hort. Sci., 14(1/2): 103-107.

5. Kaur P., Dhaliwal, M.S. and Singh, S. 2004. Genetic analysis of yield in tomato by involving genetic male sterile lines. Acta Horti. 637:155-166.

6. Kempthorne O. 1957. Introduction to Genetic Statistics. New York: John Wiley and sons, Inc; London Chapman \& Hall, Ltd.

7. Rick C.M. 1969. Origin of cultivated tomato, current status and the problem. International Bot. Cong.. pp180.

8. Singh B., Kaul S., Kumar D. and Kumar V. 2010. Combining ability for yield and its contributing characters in tomato. Indian J. Horti. 67: 50-55.

9. Singh R K and Chaudhury B D. 1985. Biometrical Methods in Quantitative Genetic Analysis. Kalayani Publishers, NewDelhi. pp. 318.

\section{How to cite this article:}

Rakesh Kumar Meena and Sanjay Kumar. 2020. Combining Ability for Yield and Quality Related Traits in Tomato (Solanum lycopersicum L.). Int.J.Curr.Microbiol.App.Sci. 9(05): 2931-2935. doi: https://doi.org/10.20546/ijcmas.2020.905.347 